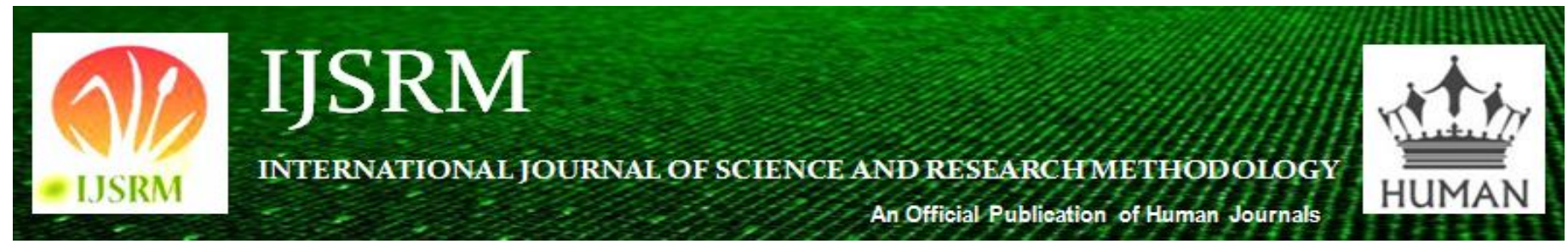

Human Journals

Review Article

January 2022 Vol.:20, Issue:3

(C) All rights are reserved by Samuel P. Abraham et al.

\title{
A Comparison Review of Mechanical Cardiopulmonary Resuscitation Vs Manual Cardiopulmonary Resuscitation
}

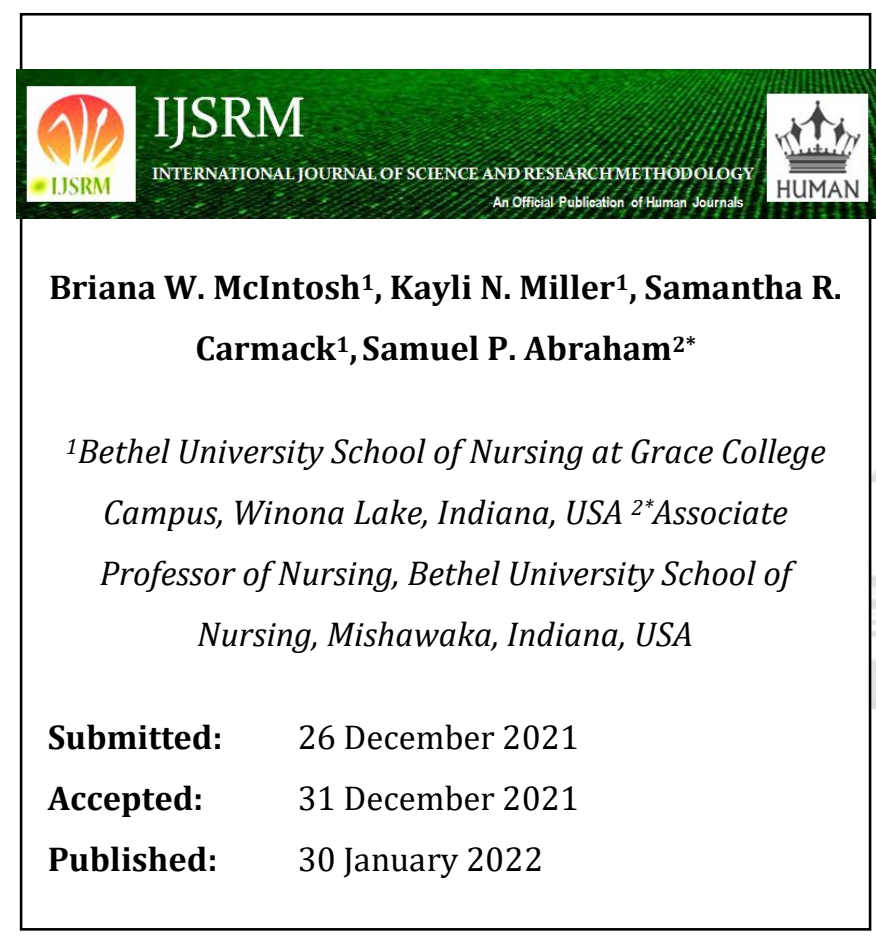
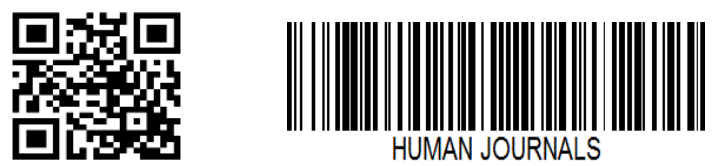

www.ijsrm.humanjournals.com
Keywords: $\mathrm{CPR}$, mechanical $\mathrm{CPR}$, chest compressions, return of spontaneous circulation, LUCAS, Zoll AutoPulse

\section{ABSTRACT}

Background: Cardiopulmonary resuscitation (CPR) is a lifesaving intervention performed by healthcare workers in the event of cardiac arrest. In recent years, many mechanical devices have been developed to perform quality compressions in place of manual chest compressions in an attempt to sustain quality CPR, as the rescuer will tire, and CPR quality will diminish. With the rise of mechanical cardiopulmonary resuscitation devices (LUCAS, Zoll AutoPulse) making mechanical CPR a realistic and practical treatment option, it now must be determined whether or not mechanical CPR leads to better outcomes in patients that have been successfully resuscitated in comparison to patients having been resuscitated by manual compressions, as is seen in traditional CPR. Purpose: The purpose of the review was to compare long-term outcomes of patients receiving mechanical cardiopulmonary resuscitation versus manual chest compressions. Method: This is a systematic review of the nursing-focused literature regarding the complications and long-term outcomes of patients receiving both manual and mechanical cardiopulmonary resuscitation. This review examined evidence-based studies of both methods to compare findings. Findings: This review found achievement of return of spontaneous circulation (ROSC), complications, and chest compression quality, that pertained to the outcomes of patients following cardiac arrest. No significant difference was noted in the outcomes. Conclusion: At this time, mechanical CPR has more benefit to the team of rescuers than to the patient receiving CPR, as it reduces the number of people needed to make resuscitation efforts and reduces loss of compression quality from fatigued rescuers. However, mechanical CPR was not found to have better patient outcomes when compared to manual CPR in cases of out-of-hospital cardiac arrests. 


\section{INTRODUCTION}

Cardiac arrest occurs when an individual's heart stops beating. Cardiopulmonary resuscitation, also commonly known as CPR, is a way of pushing on the individual's chest wall in an attempt to provide blood flow to the organs when the heart is not pumping on its own. The most common way of doing this is manual, by pressing on the individual's chest with the palms of hands in a rhythmic pattern. Quality CPR is described as uninterrupted chest compressions at a rate of 100120 beats per minute [1]. This can become a difficult task when alone, as rescuer fatigue sets in. Poole et al. [2] noted that in an attempt to improve the quality of CPR, mechanical CPR devices such as Autopulse and LUCAS were made. These machines take over and do the CPR themselves. They provide a constant set rate and depth of compressions [2].

The goal is to determine which method of cardiopulmonary resuscitation is most effective for patients while leaving patients with the fewest adverse outcomes possible. With the rise of circumferential cardiopulmonary resuscitation devices (LUCAS and AutoPulse) making circumferential CPR a realistic and practical treatment option, it now must be determined whether or not circumferential CPR leads to better outcomes in patients that have been successfully resuscitated in comparison to patients having been resuscitated by manual compressions, as is seen in traditional CPR. The purpose of the review was to compare the longterm outcomes of patients receiving circumferential cardiopulmonary resuscitation versus manual chest compressions. Question: In patients attaining return of spontaneous circulation (ROSC), how does circumferential cardiopulmonary resuscitation compared to manual CPR, affect patient long-term outcomes?

\section{BACKGROUND}

CPR is a lifesaving intervention performed on patients experiencing cardiac arrest, including the presence of lethal cardiac rhythms such as ventricular tachycardia or ventricular fibrillation. Resuscitation efforts have developed greatly to become more effective since the 1700s when Scottish surgeon William Tossach used mouth-to-mouth breaths to successfully revive a suffocated coal miner [3]. This intervention was then implemented in Paris to revive drowned victims. This led to the use of bellows being used to ventilate unresponsive victims. 
In 1874, Italian physiologist Moritz Schiff's research indicated that massaging the heart during heart surgeries can restore circulation [3]. This developed into the implementation of external chest compressions in the 1890s, but the concept did not gain traction until 1960, at which point Drs. Kouwenhoven, Safar, and Jude published their research on resuscitation that indicated chest compressions, defibrillation, and mouth-to-mouth breathing were effective resuscitation efforts. This was endorsed by the American Heart Association in 1963, and the methods were refined to become more effective. By 2005, high-quality CPR was introduced as compression to rescue breath ratio of 30:2. Today, resuscitation programs are widely available and utilized to train healthcare professionals and laypersons alike to respond appropriately in an emergency to provide lifesaving interventions early when a person experiences cardiac arrest [3].

\section{METHOD}

This is a review on the topic of the comparison of the long-term outcomes of patients receiving mechanical CPR versus manual CPR. Data was collected by literature review using Cumulative Index to Nursing and Allied Health Literature (CINAHL) and other nursing-focused databases. Google Scholar was also used to broaden the search and allow access to more diverse studies. This ensured streamlined access to information pertinent to the topic. Using the databases also gave access to peer-reviewed literature which is more reliable and higher on the pyramid of evidence. Keywords included cardiopulmonary resuscitation, mechanical cardiopulmonary resuscitation, chest compressions, and return of spontaneous circulation, LUCAS, Zoll AutoPulse, and circumferential CPR. Figure 1 displays the levels of evidence hierarchy that were used within this systematic review.

The levels of evidence are labeled level 1 through level 7 with level 1 being the highest and the best level of evidence and level 7 being the lowest quality of evidence. Fifteen studies were utilized in the initial review; however, more studies were added to complement the review. Dates of publication ranged primarily from 2016 to 2021, with one study published in 1993. The 1993 study was written about a vest that increases aortic pressures during CPR. While the research is old, the conclusions of the study support the claims of other studies. Overall, the studies chosen, fall into a higher level in the Evidence Hierarchy and are recent within the past 5 years, making the review of literature current as well as more reliable. 


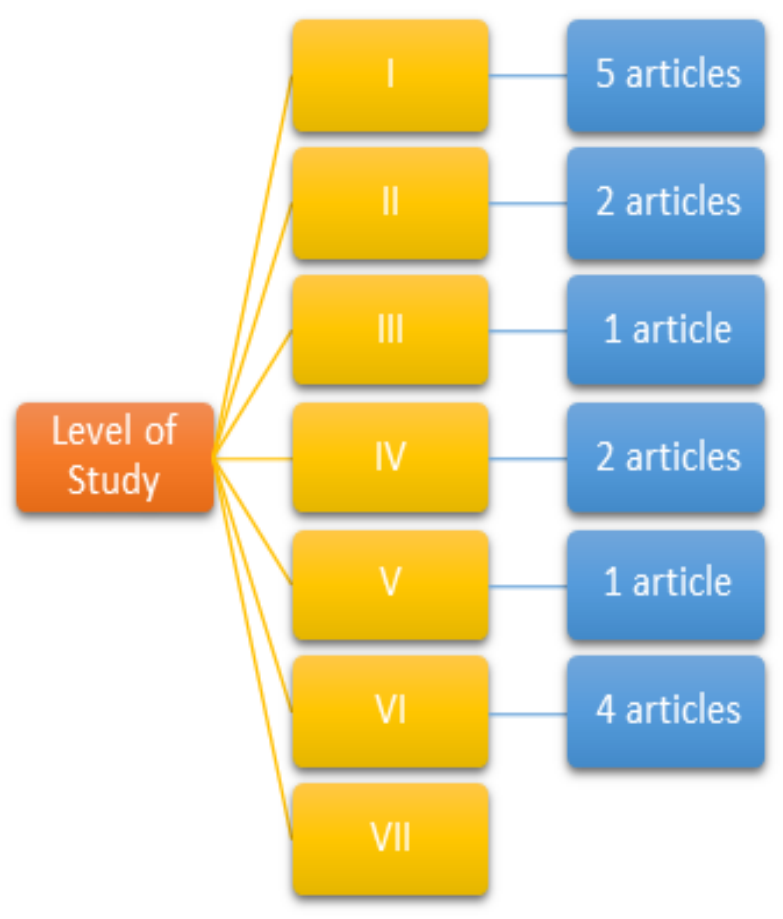

Figure 1. Studies reviewed their levels of the nursing evidence hierarchy for mechanical CPR vs. manual CPR.

\section{LITERATURE REVIEW AND FINDINGS}

Three main topics were discovered within the review. These were viewed as a priority with longterm outcomes of patients receiving mechanical cardiopulmonary resuscitation versus manual chest compressions. These are the return of spontaneous circulation, complications related to CPR, and the quality of the CPR. Each of the findings is described below.

\section{Return of Spontaneous Circulation}

Return of spontaneous circulation (ROSC) if occurs post-cardiac arrest, signifies that the heart has started to beat on its own again. ROSC signifies that the resuscitation efforts worked. The absence of the return of ROSC signifies the resuscitation efforts did not work, and the patient did not survive. Of the 15 studies that were utilized, eight of them mention ROSC, but six discuss ROSC in more detail and they are discussed here.

The study conducted on in-hospital cardiac arrests showed that mechanical CPR was associated with increased rates of the return of ROSC [4]. In another study, Hardig et al. [5] found no 


\section{www.ijsrm.humanjournals.com}

significant difference in ROSC between the usage of the mechanical CPR device LUCAS, and manual CPR.

For out-of-hospital cardiac arrests, Sheraton et a. [1] found that the use of mechanical CPR in out-of-hospital cardiac arrest did not improve ROSC. This study also mentioned previous studies that indicated mechanical CPR usage had worse outcomes for ROSC than that of manual CPR, and mechanical CPR devices should not be utilized [1]. Another study found that survival to hospital discharge was more favorable in patients who received manual CPR rather than mechanical CPR. The study also found that favorable neurological function was more likely in patients who received manual CPR [6].

Suh et al. [7] conducted a study on an end-tidal CO2-guided automated robot CPR system. This system finds the best compression position for CPR for both manual and mechanical CPR systems. This study was conducted on swine models and found that there was no significant difference in ROSC between mechanical CPR and manual CPR when using the end-tidal CO2guided automated robot CPR system [7].

One literature review that was included showed very mixed results for ROSC. In a review, Wang and Brooks [8] included eight studies. Three studies showed a benefit in mechanical CPR over manual CPR, four studies showed no significant difference between the use of mechanical CPR and manual CPR, and one study showed a decrease in ROSC when using mechanical CPR. These studies show mixed results and indicate that further research is needed.

\section{Complications Related to CPR}

One study result suggests that mechanical chest compressions do not increase the incidence and severity of CPR-related injury in comparison with manual methods despite significantly longer CPR duration [9]. However, another study [10] revealed that chest compressions often leave iatrogenic injuries to the victim, such as rib fractures, visceral injuries, and soft tissue damage(see Figure 2). Seven of the studies reviewed complications of mechanical CPR using devices such as the LUCAS, the Zoll AutoPulse, and circumferential CPR vests, as well as the complications of manual CPR. Both methods of CPR left patients with iatrogenic injuries, but the nature and severity of the injuries varied by case. In manual CPR, the most common 


\section{www.ijsrm.humanjournals.com}

complication was rib fractures in the midclavicular line, which were present in $26.1 \%$ of cases. This is followed by soft tissue injuries in $18.2 \%$ of cases. These injuries were found to be more severe in cases with multiple rescuers, all of which were categorized as moderate to severe, never mild. In single-person resuscitation, mild injuries were present in $23.8 \%$ of cases, moderate injuries were found in $23.8 \%$ of cases, and severe injuries were found in $28.6 \%$ of cases [10]. Of resuscitations studied, $96.6 \%$ were performed by specialized medical staff trained in resuscitation efforts.

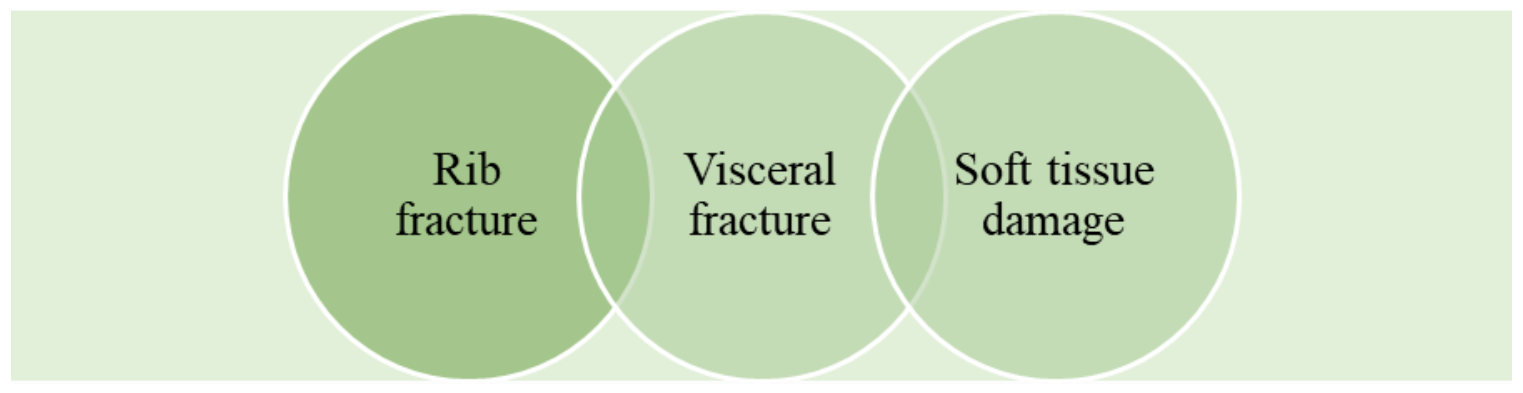

Figure 2. Chest compressions in manual CPR can cause iatrogenic injuries.

In another study, Ho et al [11] followed the case of a 68-year-old male who experienced an aortic dissection that was ruled to be an iatrogenic injury following the use of a LUCAS device to deliver mechanical CPR. No manual CPR was performed. In studying this outcome and comparing it to other complications, it was noted that the use of the LUCAS device correlated with an increased number of skeletal and soft tissue injuries, which has been attributed to more powerful compressions and placement of the suction cup [11].

Elison et al. [12] followed a 46-year-old male receiving CPR from a LUCAS device after experiencing ventricular fibrillation that did not respond to defibrillation that progressed to pulseless electrical activity. A sternotomy revealed a laceration of the left ventricle that resulted in a hemorrhagic hemothorax. Following the repair of the left ventricle, the patient's condition progressed to multisystem organ failure, and he ultimately expired from the injuries. It was determined that the suction cup on the LUCAS device was positioned directly on top of the left ventricle, causing the laceration [12]. 


\section{www.ijsrm.humanjournals.com}

As seen in Figure 3, the most common life-threatening injury discovered with mechanical CPR is damage to visceral organs, including large vessels and vertebrae [13]. This finding considers the LUCAS, Zoll AutoPulse, and also a Phillips MRx for manual chest compressions. None of the reported cases resulted in lethal injuries beyond the cause of cardiac arrest, and injuries were found to be more severe at varying degrees when CPR was performed by more than one rescuer, as opposed to injuries when CPR was performed by a single person [13].

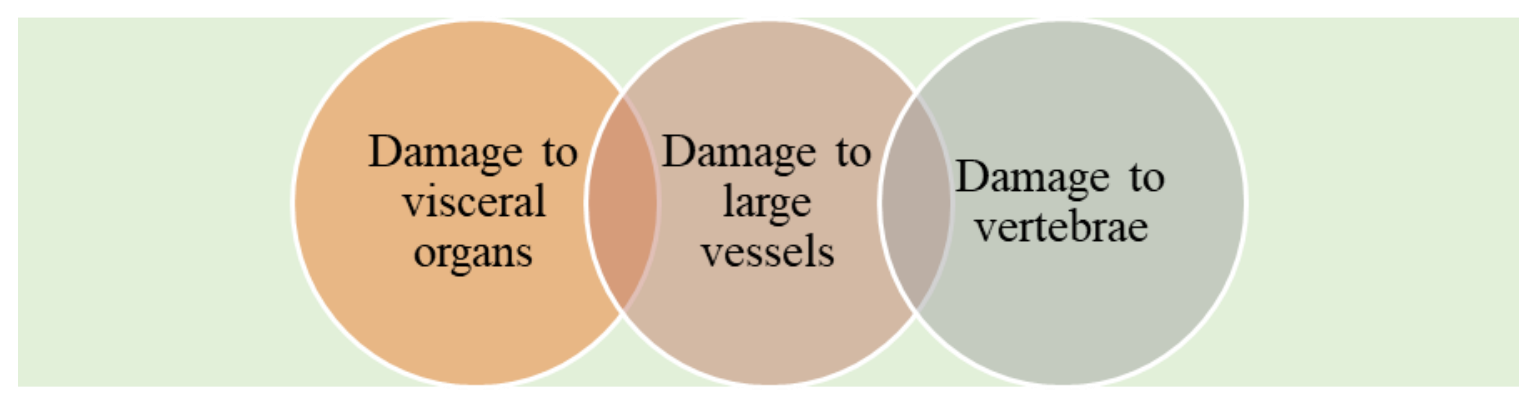

Figure 3. Common life-threatening injury with mechanical CPR.

In contrast, Milling et al. [14] discovered that five of 50 cases studied in their research induced iatrogenic injuries from mechanical CPR that would have been life-threatening if the patient had survived the arrest. Of those studied, 11 patients had visceral injuries, four had abdominal injuries, and 23 experienced only thoracic injuries. Only 12 patients studied had no injury following mechanical CPR [14].

Canakci et al. [15] followed the cases of patients experiencing ROSC following a cardiac arrest to compare methods of CPR when used in in-hospital arrests. Manual chest compressions were performed in 131 cases, and 47 cases utilized a mechanical CPR device. Computed tomographic (CT) findings following the achievement of ROSC displayed no significant differences between the two methods. Complications of both included lung contusion, rib fracture, and pneumothorax, with no statistically significant changes in incidence. One sternum fracture and one hemothorax were exclusive to the manual CPR group, and neither group had any incidences of aortic dissection, solid organ injury, or cardiac injury [15].

Because ROSC is rarely achieved after 20 minutes of manual chest compressions, Halperin et al. [16] published a study in which a circumferential bladder-containing vest was applied to perform mechanical compressions after 20 minutes of manual CPR. ROSC was achieved in four of 29 
patients after application of the vest, but none survived to be discharged from the hospital [16]. Another study found that mechanical compared to manual chest compression delivered a more rapid compression and decompression of the cardiac structures at an adequate rate, with the broader inward-outward movement of the ventricular walls suggesting greater emptying and filling of the ventricles [17].

\section{The Quality of CPR}

The American Heart Association [18] propagates the quality of cardiopulmonary resuscitation can be determined by the rate and depth of compressions. The American Heart Association has determined that CPR compressions should be at a rate of 100-120 bpm and a depth of 2" in adults. The following studies have addressed the quality of CPR during manual CPR as compared to mechanical CPR [18].

In a study, Obermaier et al. [19] addressed the quality of CPR. The study was conducted to determine whether the intended use of mechanical CPR is played out in practice. The intended use of mechanical CPR is to administer consistent effective compressions without the human fatigue aspect. The conclusion of the study explained three studies on mechanical CPR. One was a randomized assessment, and the others were retrospective registry analyses. As shown in Figure 4, it was unclear whether one was more effective than the other when it came to improved outcomes [19]. They concluded that between three different studies there was not a statistically significant difference between the qualities in the use of the LUCAS versus manual compressions. This could have been due to the location where the mechanical device was used in the field rather than a controlled hospital environment. Part of the study indicated the use by paramedics. Ambulance transport are not known to be smooth. The quick turns and unpredictable road conditions affect the movement of the vehicle. This in turn could affect the quality of CPR administered in the field. In which case, there would not be a difference between the mechanical and manual CPR [19]. 


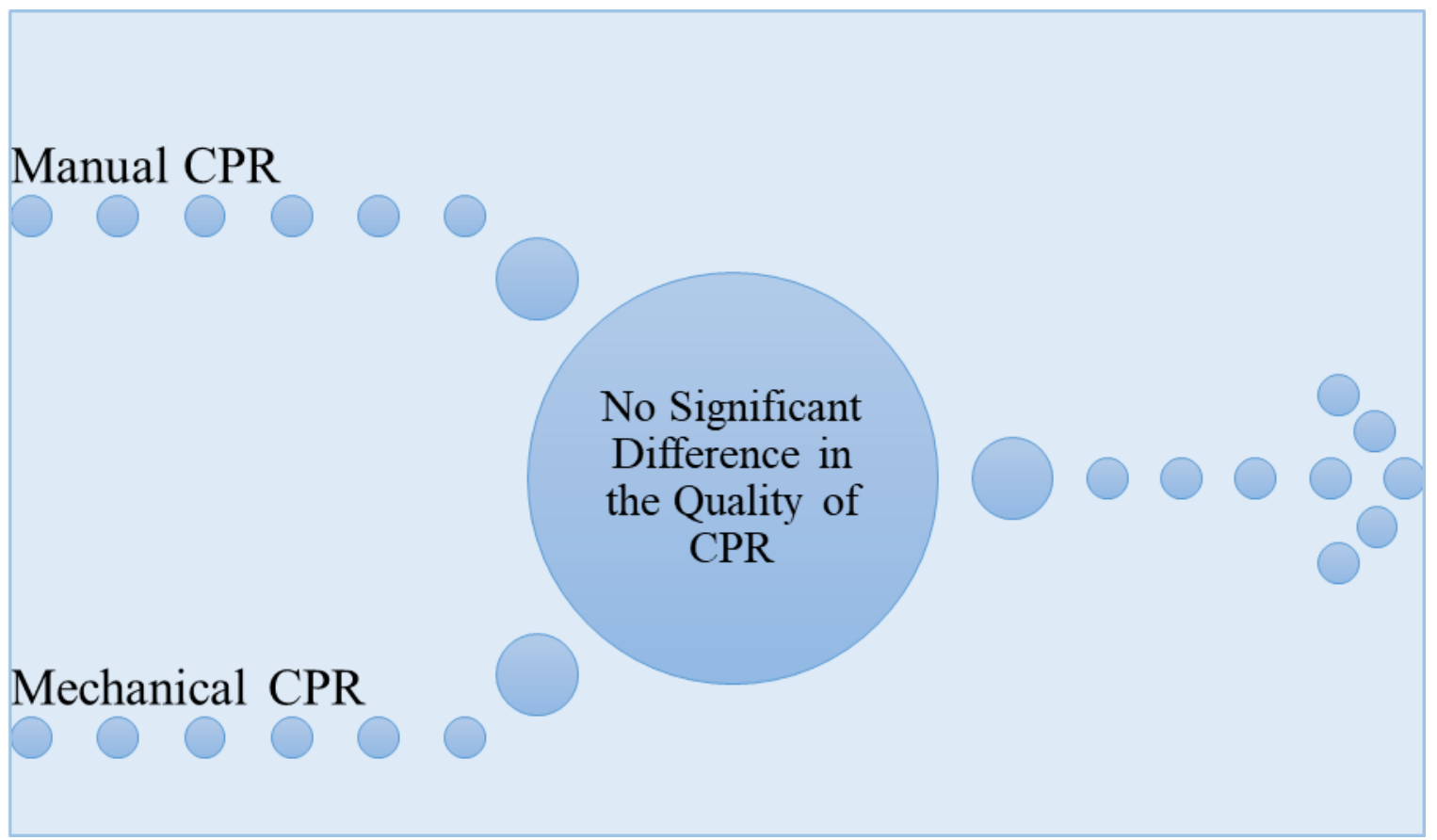

Figure 4. The quality of CPR does not seem to be significant in both methods and the outcome is similar.

The synthesis of presented evidence does not support that mechanical chest compression with a LUCAS device improves clinical outcomes in out-of-hospital cardiac patients compared with manual chest compression [20]. Comparisons of mechanical and manual chest compressions terms of survival rates and favorable neurological outcomes showed no significant differences [21]. In the Sheraton et al [1] study, CPR effectiveness was compared to CPR with mechanical devices. The analysis concluded that mechanical devices do have a place in non-ideal resuscitations where early initiation of quality CPR has not been possible [1]. This means in terms of quality there is no advantage between the devices, however, the advantage identified is in terms of convenience in times where assistance is needed, and help is not readily available.

Uil et al. [22] suggested that mechanical CPR devices such as LUCAS and AutoPulse might help ensure good-quality prolonged CPR. Several ongoing randomized trials protocol led to routine mechanical CPR during immediate transport to an ECPR capable emergency department. The introduction of mechanical devices came intending to ensure good-quality prolonged CPR [22]. The specific study being analyzed focused specifically on injuries induced by CPR devices such as LUCAS and AUTOPULSE. The quality of CPR is discussed because injuries may be 


\section{www.ijsrm.humanjournals.com}

sustained by the devices. The consistent prolonged use of LUCAS or AutoPulse introduces injuries due to the intense quality pressure. Cardiopulmonary resuscitation comes with specific risks whether it is completed manually or mechanically [22]. Three major findings of the study are shown in Figure 5.

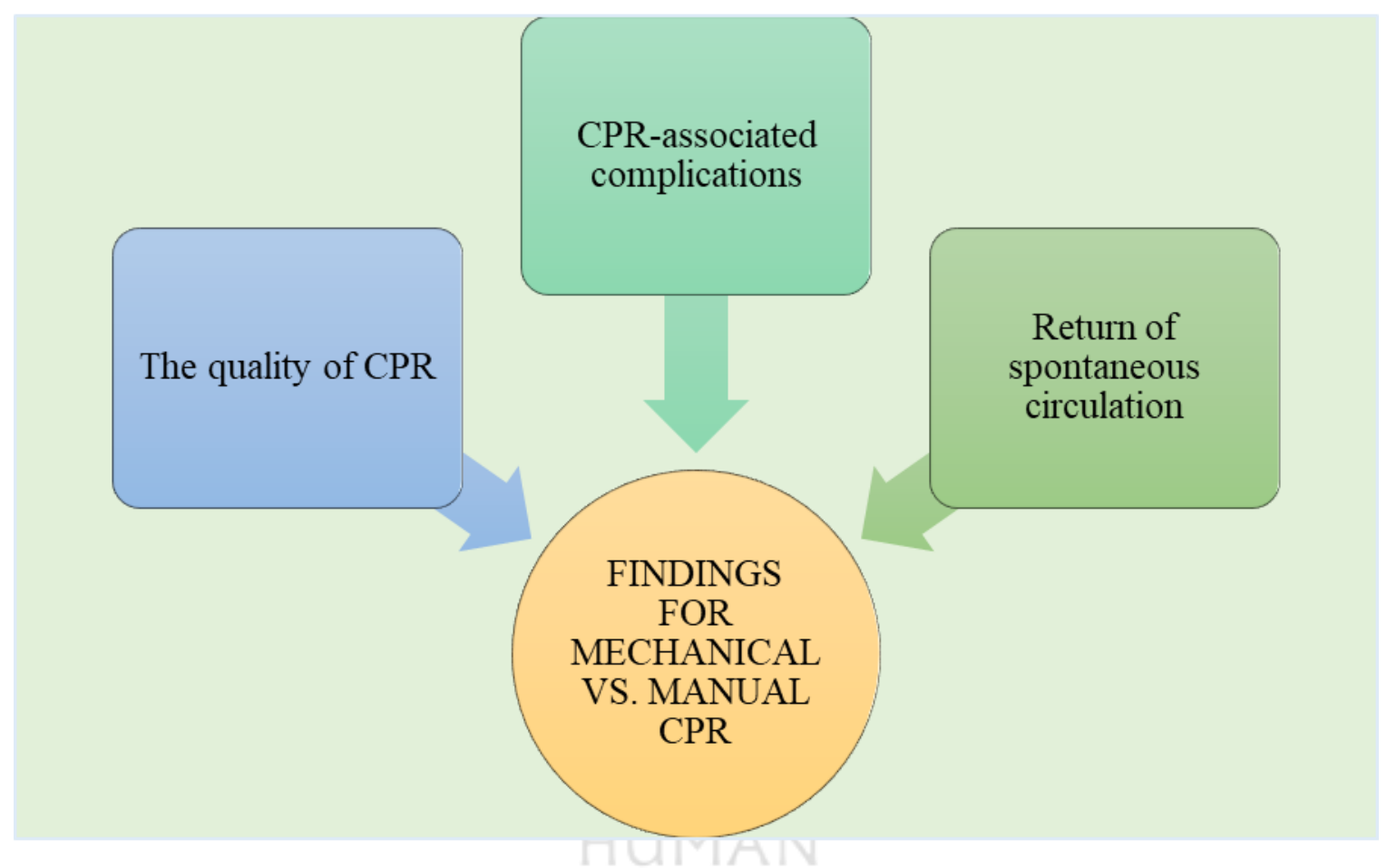

Figure 5. Findings for mechanical vs. manual CPR.

\section{DISCUSSION}

Most studies found that there was not a significant difference between the long-term effects of mechanical CPR and manual CPR in cases of cardiac arrest. Mechanical CPR devices such as Autopulse and LUCAS were found to be reasonable alternatives to manual CPR when utilized properly, especially in cases where high-quality manual CPR may be more difficult to achieve. However, not enough information was found to recommend mechanical CPR over manual.

One study found mechanical CPR to have increased rates of ROSC within the hospital setting [4]. This study specifically pointed to mechanical CPR having better outcomes. Four other studies found there to be no significant difference between mechanical and manual CPR. Two of those studies found there to be no significant difference between the two in terms of ROSC [5,7]. 
The other two studies found there to be no significant difference in the quality of the CPR the patients received $[19,20]$.

One study mentioned mechanical CPR having worse outcomes. It indicated that mechanical CPR usage had worse outcomes for ROSC than that of manual CPR, and mechanical CPR devices should not be utilized [1]. However, Buckler et al. [6] found manual CPR to have better neurological outcomes. Lastly, one literature review had mixed findings [8]. Of the eight studies included in their review, three showed a benefit of mechanical CPR over the manual. Four showed no significant difference between the two, and lastly one showed a decrease in ROSC with the usage of mechanical CPR [8].

Recent studies, ones within the past five years, were chosen because they included the most recent standards and information. CPR in the emergency department is more controlled because there is access to monitoring and supplies. In the field, external factors such as bumpy roads or the movement of the transport vehicle also affect outcomes. Current studies follow the immediate results and effects of CPR not necessarily the long-term effects. While some of these long-term effects can be inferred based on the results, for example, death from unsuccessful CPR, more studies should be done to research more specifically.

\section{Recommendations}

Multiple studies have already been conducted on out-of-hospital cardiac arrests and found that there is no significant difference between the outcomes of patients who received mechanical CPR versus the patients who received manual CPR. It is recommended for more studies to be conducted on the use of mechanical CPR in hospital settings.

While there may not be a significant difference in long-term effects, mechanical CPR devices should become more widely accessible. Using these devices cuts down on the need for many staff members. This is beneficial to the hospital because staffing can be supplied in areas where currently it is lacking. The patient is receiving compressions that consistently meet the standards of the AHA. Training staff on this machine would also be required. The machine does not have the human fatigue element; however, staff needs to know how to set it up and maintain it. 


\section{www.ijsrm.humanjournals.com}

Educating staff members on the importance of safety with the devices could help with the complications currently tied to the use of mechanical CPR. The proper use of the machines minimizes the risk of user complications. Staff should also continue to be educated on proper manual CPR techniques. Basic life support and advanced life support are current AHA certifications required in the in-hospital healthcare environment. This should be continued to improve patient outcomes.

\section{CONCLUSION}

Adverse patient outcomes can occur in both manual and mechanical CPR. Both manual and mechanical CPR has their strengths and weaknesses when utilized in different settings. Mechanical CPR was not found to have better patient outcomes when compared to manual CPR in cases of out-of-hospital cardiac arrests. However, mechanical CPR may be more beneficial in cases where there are fewer people available, rescuer fatigue, and in cases where there is a lack of resources. Mechanical CPR may improve the quality of CPR, but it has not been found to improve ROSC outcomes.

\section{REFERENCES}

1. Sheraton, M., Columbus, J., Surani, S., Chopra, R., \& Kashyap, R. (2021). Effectiveness of mechanical chest compression devices over manual cardiopulmonary resuscitation: A systematic review with meta-analysis and trial sequential analysis. Western Journal of Emergency Medicine, 22(4), 810-819. https://doi.org/10.5811/westjem.2021.3.50932

2. Poole, K., Couper, K., Smyth, M. A., Yeung, J. \& Perkins, G. D. (2018). Mechanical CPR: Who? When? How? Critical Care, 22, 140. https://doi.org/10.1186/s13054-018-2059-0

3. American Heart Association. (n.d.). History of CPR. cpr.heart.org. Retrieved November 21, 2021, from https://cpr.heart.org/en/resources/history-of-cpr.

4. Crowley, C. P., Wan, E. S., Salciccioli, J. D., \& Kim, E. (2020). The use of mechanical cardiopulmonary resuscitation may be associated with improved outcomes over manual cardiopulmonary resuscitation during inhospital cardiac arrests. Critical Care Explorations, 2(11), e0261. https://doi.org/10.1097/CCE.0000000000000261

5. Hardig, B. M., Lindgren, E., Östlund, O., Herlitz, J., Karlsten, R., Rubertsson, S. (2017). Outcome among VF/VT patients in the LINC (LUCAS IN cardiac arrest) trial: A randomised, controlled trial. Resuscitation. doi: 10.1016/j.resuscitation.2017.04.005

6. Buckler, D. G., Burke R.V., Naim M. Y., MacPherson A., Bradley R. N, ... Rossano R.W. (2016). Association of mechanical cardiopulmonary resuscitation device use with cardiac arrest outcomes. Circulation,134(25), 21312133. doi:10.1161/CIRCULATIONAHA.116.026053.

7. Suh, J. G., Park, J., Lee, C.J., Na, S.H., Kwon, W. Y., Kim, S.K., Kim, T... You, M.K. (2018). End-Tidal CO2guided automated robot CPR system in the pig. preliminary communication. Resuscitation, 127:119-24. doi: 10.1016/j.resuscitation.2018.04.011.

8. Wang, P. L., Brooks, S. C. (2018). Mechanical versus manual chest compressions for cardiac arrest. Cochrane Database of Systematic Reviews, 8, CD007260. doi: 10.1002/14651858.CD007260.pub4 


\section{www.ijsrm.humanjournals.com}

9. Karasek, J., Blankova, A., Doubková, A., Pitasova, T., Nahalka, D., Bartes, T., Hladik, J., Adamek, T., Jirasek, T., Polasek, R., \& Ostadal, P. (2021). The comparison of cardiopulmonary resuscitation-related trauma: Mechanical versus manual chest compressions. Forensic Science International, 323, 110812-110812. https://doi.org/10.1016/j.forsciint.2021.110812

10. Deliliga, A., Chatzinikolaou, F., Koutsoukis, D. et al. (2019) Cardiopulmonary resuscitation (CPR) complications encountered in forensic autopsy cases. BMC Emerg Med, 19, 23 https://doi.org/10.1186/s12873-0190234-5

11. Ho, K., Kopriva, D., \&Dehghani, P. (2020). A case of an aortic dissection after mechanical chest compression by LUCAS. JACC Case Reports, 2(12). doi: 10.1016/j.jaccas.2020.06.033.

12. Elison, D., Don, C., \& Nakamura, K. (2019). Coronary artery and right ventricular perforation due to mechanical CPR trauma. JACC Journals. https://www.jacc.org/doi/full/10.1016/j.jaccas.2019.08.007

13. Koster, R. W., Beenen, L. F., van der Boom, E. B., Spijkerboer, A. M., Tepaske, R., van der Wal, A. C., ... Tijssen, J. G. (2017). Safety of mechanical chest compression devices AutoPulse and LUCAS in cardiac arrest: A randomized clinical trial for non-inferiority. European Heart Journal, 38(40). doi: 10.1093/eurheartj/ehx318

14. Milling, L., Mikkelsen, S., Astrup, B. S. (2020). Characteristics of mechanical CPR-related injuries: A case series. Journal of Forensic and Legal Medicine: 70(2). doi: 10.1016/j.jflm.2020.101918

15. Canakci, M. E., Bagceci, K. P., Acar, N., Ozakin, E., Baloglu, K. F., Kuas, C... \& Karakılı̨̧, M. E. (2021). Computed tomographic findings of injuries after mechanical and manual resuscitation: A retrospective study. Cureus, 13(5), e15131. https://doi.org/10.7759/cureus.15131

16. Halperin, H. R., Tsitlik J E., Gelfand M., Weisfeldt M. L., Gruben K. G., Levin H.R., Guerci A. D. (1993.) A preliminary study of cardiopulmonary resuscitation by circumferential compression of the chest with use of a pneumatic vest. New England Journal of Medicine, 329(11):762-68. doi: 10.1056/NEJM199309093291104.

17. Colombo, R., Fossali, T., Ottolina, D., Borghi, B., Bergomi, P., Ballone, E., Rech, R., Castelli, A., \& Catena, E. (2019). Kinetics of manual and automated mechanical chest compressions. Resuscitation, 145, 70-74. https://doi.org/10.1016/j.resuscitation.2019.10.009

18. American Heart Association. [2021]. CPR \& First Aid Emergency Cardiovascular Care, Retrieved from https://cpr.heart.org/en/resources/what-is-cpr

19. Obermaier, M., Zimmermann, J. B., Popp, E., et al. (2021). Automated mechanical cardiopulmonary resuscitation devices versus manual chest compressions in the treatment of cardiac arrest: Protocol of a systematic review and meta-analysis comparing machine to human. BMJ Open, 11: e042062. doi: 10.1136/bmjopen-2020042062

20. Liu, M., Shuai, Z., Ai, J., Tang, K., Liu, H., Zheng, J., Gou, J., \& Lv, Z. (2019). Mechanical chest compression with LUCAS device does not improve clinical outcome in out-of-hospital cardiac arrest patients: A systematic review and meta-analysis. Medicine (Baltimore), 98(44), e17550-e17550. https://doi.org/10.1097/MD.0000000000017550

21. Halhalli, H. C., Şanc1, E., \& Uslu, T. (2020). The comparison of manual and mechanical chest compression on survival and long-term neurological outcome of nontraumatic out-of-hospital cardiac arrest patients. The Journal of Emergency Medicine, 59(5), 680-686. https://doi.org/10.1016/j.jemermed.2020.06.010

22. Uil, C. A. den, Bonnes J.L., \& Brouwer M.A. (2018). Mechanical CPR in refractory cardiac arrest may be practical, but injuries should be monitored: A concise Meta-analysis. Resuscitation, 122, 5-6. doi: 10.1016/j.resuscitation.2017.05.022. 
www.ijsrm.humanjournals.com

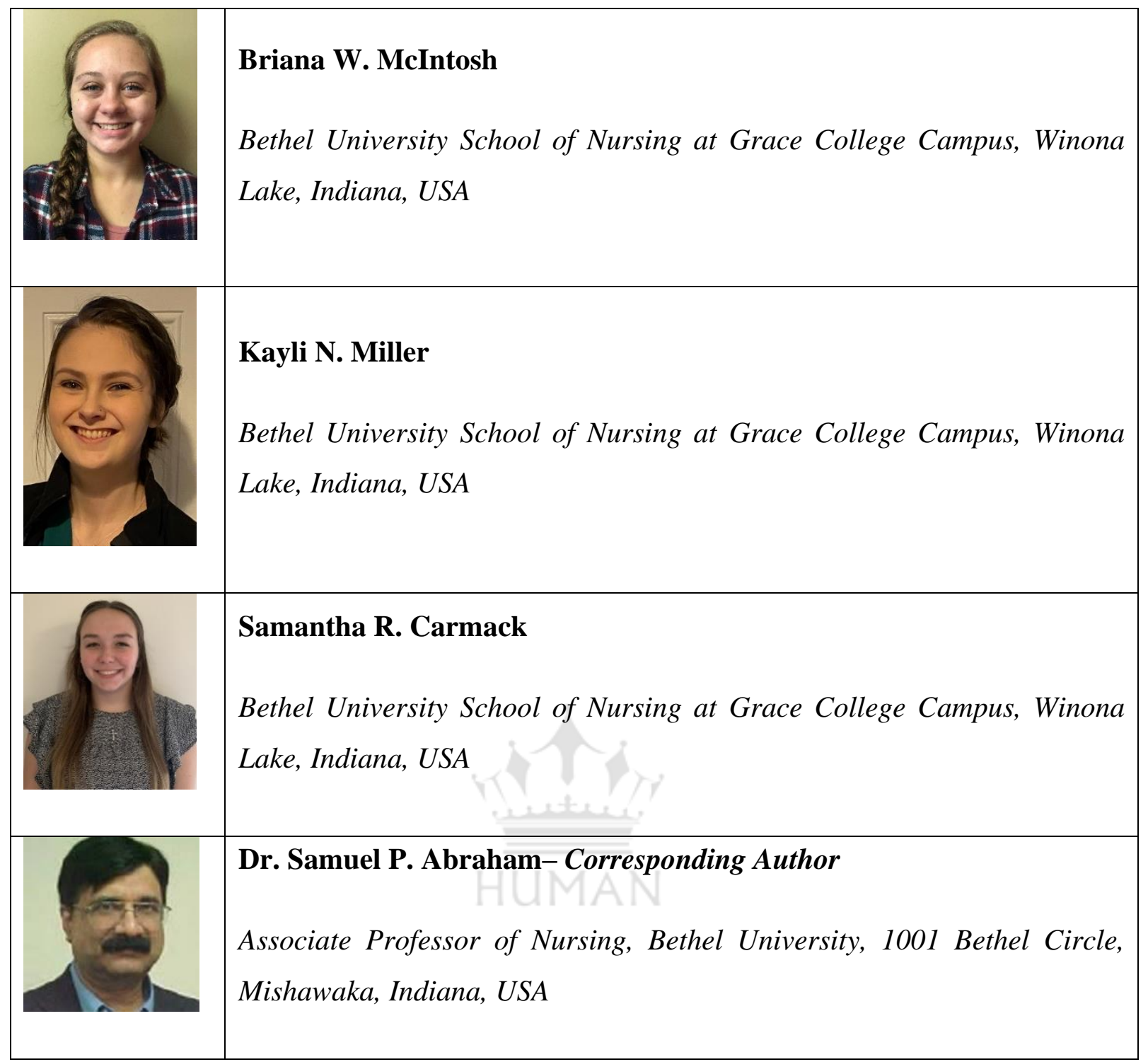

